\title{
Lung cGMP release subsequent to NO inhalation in pulmonary hypertension: responders versus nonresponders
}

\author{
H.A. Ghofrani, R. Wiedemann, F. Rose, N. Weissmann, R.T. Schermuly, K. Quanz, F. Grimminger, \\ W. Seeger, H. Olschewski
}

Lung cGMP release subsequent to NO inhalation in pulmonary hypertension: responders versus nonresponders. H.A. Ghofrani, R. Wiedemann, $F$. Rose, $N$. Weissmann, R.T. Schermuly, K. Quanz, F. Grimminger, W. Seeger, H. Olschewski. (C) ERS Journals Ltd 2002.

ABSTRACT: Inhalation of nitric oxide (NO) is widely employed for the assessment of pulmonary vasoresponsiveness in pulmonary hypertension $(\mathbf{P H})$. However, the reasons for the huge differences in vascular reactivity to NO between patients are unknown, and the role of NO-induced cyclic guanosine monophosphate (cGMP) is unclear.

Twenty patients with severe precapillary $\mathrm{PH}$ were investigated. Thirty-six SwanGanz catheter investigations were performed and the study subjects were tested for responses to NO inhalation. This included an assessment of pulmonary and systemic arterial plasma cGMP and atrial natriuretic peptide (ANP) levels.

A significant NO response (pulmonary vascular resistance (PVR) decrease $>\mathbf{2 0} \%$ ) was noted in nine of 20 patients $(\mathbf{4 5 \%})$ during the first catheterization. A highly significant correlation between baseline plasma cGMP and ANP levels with PVR was observed $(r=0.62$ and $r=0.66$, respectively; $p<0.0001)$. In response to NO, systemic and mixed venous cGMP levels increased from $13.9 \pm 1.28 \mathrm{nM}$ and $12.75 \pm 0.99 \mathrm{nM}$ to $79.23 \pm 4.99 \mathrm{nM}$ and $55.25 \pm 4.41 \mathrm{nM}(\mathrm{p}<0.001)$, respectively, accompanied by the appearance of a marked transpulmonary cGMP gradient. Although in the responder group ANP levels were significantly reduced after NO inhalation, no significant correlation was observed to the extent of PVR reduction. The magnitude of the NOelicited cGMP response did not discriminate between haemodynamic responders and nonresponders.

This study concludes that plasma cyclic guanosine monophosphate levels are significantly correlated with the severity of disease in pulmonary arterial hypertension. Nitric oxide inhalation provokes a prompt increase in cyclic guanosine monophosphate secretion, but the magnitude of this release is not linked with a decrease in pulmonary vascular resistance.

Eur Respir J 2002; 19: 664-671.
Dept of Internal Medicine, Medical Clinic II, University Hospital, JustusLiebig-University, Giessen, Germany.

Correspondence: H.A. Ghofrani

Dept of Internal Medicine

Klinikstrasse 36

35392 Giessen

Germany

Fax: 496419942419

E-mail: Ardeschir.Ghofrani@innere.med. uni-giessen.de

Keywords: Catheterization

nitric oxide

pulmonary hypertension

second messenger

Swan-Ganz

Received: July 52001

Accepted after revision December 21 2001

This work was supported by a grant from the German Research Foundation.
Primary pulmonary hypertension $(\mathrm{PPH})$ is a rare disease of unknown aetiology, which is associated with a poor prognosis $[1,2]$. Only a minority $(\sim 25 \%)$ of these patients display an acute strong pulmonary vasodilatory response to calcium channel blockers, and long-term administration of these drugs in high doses has been shown to improve survival in this subgroup [3]. In contrast, such pharmacological therapy may be hazardous in haemodynamic "nonresponders" [4] and is therefore discouraged. Instead of directly testing with calcium channel blockers during right heart catheterization, vasoreactivity testing with inhaled nitric oxide (NO), being advantageous due its very short half-life and its selectivity for the pulmonary vasculature, is a widely-accepted procedure for detecting patients who are eligible for high-dose calcium channel blocker therapy [4-6].

Cyclic guanosine monophosphate (cGMP) is recognized as the predominant intracellular second messenger of NO, stemming from activation of the soluble guanylate cyclase [7, 8]. Measurements of plasma cGMP levels during inhalation of NO have been performed in rats and humans $[9,10]$. In patients who were mechanically ventilated due to acute lung failure, NO inhalation resulted in a significant elevation of plasma cGMP levels. However, there was no correlation between the individual cGMP response and the improvement in ventilation/perfusion matching, and, therefore, arterial oxygenation, in these patients [10]. Levels of urinary cGMP were shown to correlate with the severity of the disease in patients with pph [11]. This, however, was ascribed to increased levels of atrial natriuretic peptides (ANP), which, like NO, activate guanylate cyclase activity. ANP is a potent pulmonary vasodilator [12] and possesses considerable antiproliferative [13-15] and diuretic properties. These features contrast with the pathophysiological abnormalities encountered in PPH and secondary pulmonary hypertension (SPH), such as pulmonary vasoconstriction, pulmonary artery remodelling with 
proliferation of various cell types, and oedema formation. The effect of ANP is directly antagonistic to the renin angiotensin-aldosterone system [16] and to endothelin, the latter of which has been shown to be increased in pulmonary hypertension $(\mathrm{PH})$.

The present study included 20 patients with severe precapillary PH. They were classified as having PPH, pulmonary arterial hypertension associated with collagen vascular disease, and $\mathrm{PH}$ secondary to interstitial lung disease, according to the new diagnostic classification [17]. Measurements of pulmonary and systemic arterial levels of cGMP at baseline and in response to NO inhalation were performed. Correlation analysis between the individual cGMP values and haemodynamic data was carried out to examine whether: 1) plasma cGMP levels are linked with the severity of $\mathrm{PH}$; 2) an increase in the transpulmonary gradient of cGMP in response to NO inhalation, suggesting lung origin of this second messenger, may be demonstrated in both PPH and SPH; and 3) the individual cGMP response to NO inhalation is linked with the pulmonary vasodilatory response to this agent, as such a finding might explain the appearance of responders and nonresponders and might then serve as a biochemical tool for noninvasive identification of responders.

\section{Materials and methods}

\section{Materials}

The pulmonary arterial catheter $(7.5 \mathrm{~F} 754 \mathrm{H}$; Baxter, Deerfield, IL, USA) and femoral artery catheters (Vygon, Aachen, Germany) were inserted using standard techniques. Haemodynamic parameters and electrocardiogram (ECG), as well as arterial and mixed venous oxygen saturation, were monitored continuously using the Explorer (Baxter). This device was also used for measurements of cardiac output and right ventricular ejection fraction (thermodilution method).

\section{Study protocol and measurements}

Patient groups. Fourteen patients with $\mathrm{PPH}$, four patients with $\mathrm{PH}$ secondary to chronic lung fibrosis (interstitial lung disease), and two patients with pulmonary arterial hypertension associated with mixed collagen vascular disease (with a mean pulmonary artery pressure of $\geqslant 30 \mathrm{mmHg}$ ) were included in this study. Written informed consent was obtained from all patients. The patients were divided into two groups, depending on their haemodynamic responsiveness to NO inhalation (responders defined by a pulmonary vascular resistance (PVR) reduction of $\geqslant 20 \%$ ). Sixteen patients were tested for a second time after $1 \mathrm{yr}$. With regard to the first test only, six of the fourteen PPH patients were responders $(42 \%)$ and three of the six patients with sph $(50 \%)$ met the response criterium. This study was approved by the local ethics committee. Diagnostic procedures preceding patient recruitment included routine laboratory and immunological analysis, chest radiography, lung function testing, carbon monoxide diffusion testing, echocardiography, high-resolution computed tomography scan of the lung, and perfusion scintigraphy. In all cases with suspicious perfusion scintigraphy, a spiral computed tomography scan and/or pulmonary angiography was performed to exclude pulmonary embolism. Basic clinical characteristics, haemodynamic data and concomitant chronic medication of the patients are given in table 1 .

Test procedure. In all patients, catheter testing was clinically indicated. Testing was performed in the intensive care unit of the Internal Medicine Dept of the University of Giessen. Patients received a Swan-Ganz catheter via the internal jugular vein and a femoral arterial line after local anaesthesia. Measurements, including haemodynamic parameters, systemic and pulmonary arterial blood sampling for blood gas, cGMP and ANP analysis, were performed at baseline and during the last $2 \mathrm{~min}$ of NO application. Pulmonary haemodynamics were measured and calculated as described previously [18]. NO was titrated individually, administered via a side port connected to the inspiration loop of the inhalation tube system. The upper limit of NO was reached: 1) when no additional haemodynamic effect was achieved despite increasing the dose; 2 ) at the onset of side-effects (i.e. decrease in arterial oxygen saturation $>2 \%$ ); or 3 ) at a maximum dose of 25 parts per million ( $\mathrm{ppm}$ ) NO in the expiration loop. The mean NO dosage, averaged over all patients, was $15 \pm 6 \mathrm{ppm}$, with no significant difference between patients with PPH and SPH.

Sample collection. Blood samples for plasma cGMP and ANP were drawn from the pulmonary and systemic artery simultaneously, after complete haemodynamic measurements. Blood was drawn into chilled tubes containing ethylenediamine tetraacetic acid (EDTA) and 1,000 kallikrein inhibiting unit $(\mathrm{KIU}) \cdot \mathrm{mL}^{-1}$ aprotinin (Trasylol $\AA$, Bayer $\mathrm{AG}$, Leverkusen, Germany), and placed immediately on ice. Within $2 \mathrm{~h}$, the tubes were centrifuged at $2,100 \times g$ for $15 \mathrm{~min}$ at $4{ }^{\circ} \mathrm{C}$, and then plasma was transferred to polypropylene tubes and stored at $-80^{\circ} \mathrm{C}$. Measurements of plasma ANP and cGMP concentrations were carried out in duplicate by radioimmunoassay (RIA) (ANP RIA: Nichols, Bad Nauheim, Germany; cGMP RIA: Coulter-Immunotech, Hamburg, Germany). One assay kit was used for all ANP measurements in one catheter investigation. The mean inter-assay variations of the ANP RIA and the cGMP RIA were $\sim 34 \%$ and $\sim 5 \%$, respectively, as determined by repetitive measurements of control probes. These differences were compensated for by correction factors derived from a standard probe.

Statistics. All data are given as mean \pm SEM. Unpaired two tailed t-tests were employed to determine the differences between the baseline parameters of the responders and nonresponders, and the differences in their responsiveness to $\mathrm{NO}$ inhalation. The same test was applied to search for statistical differences between baseline parameters and differences in 


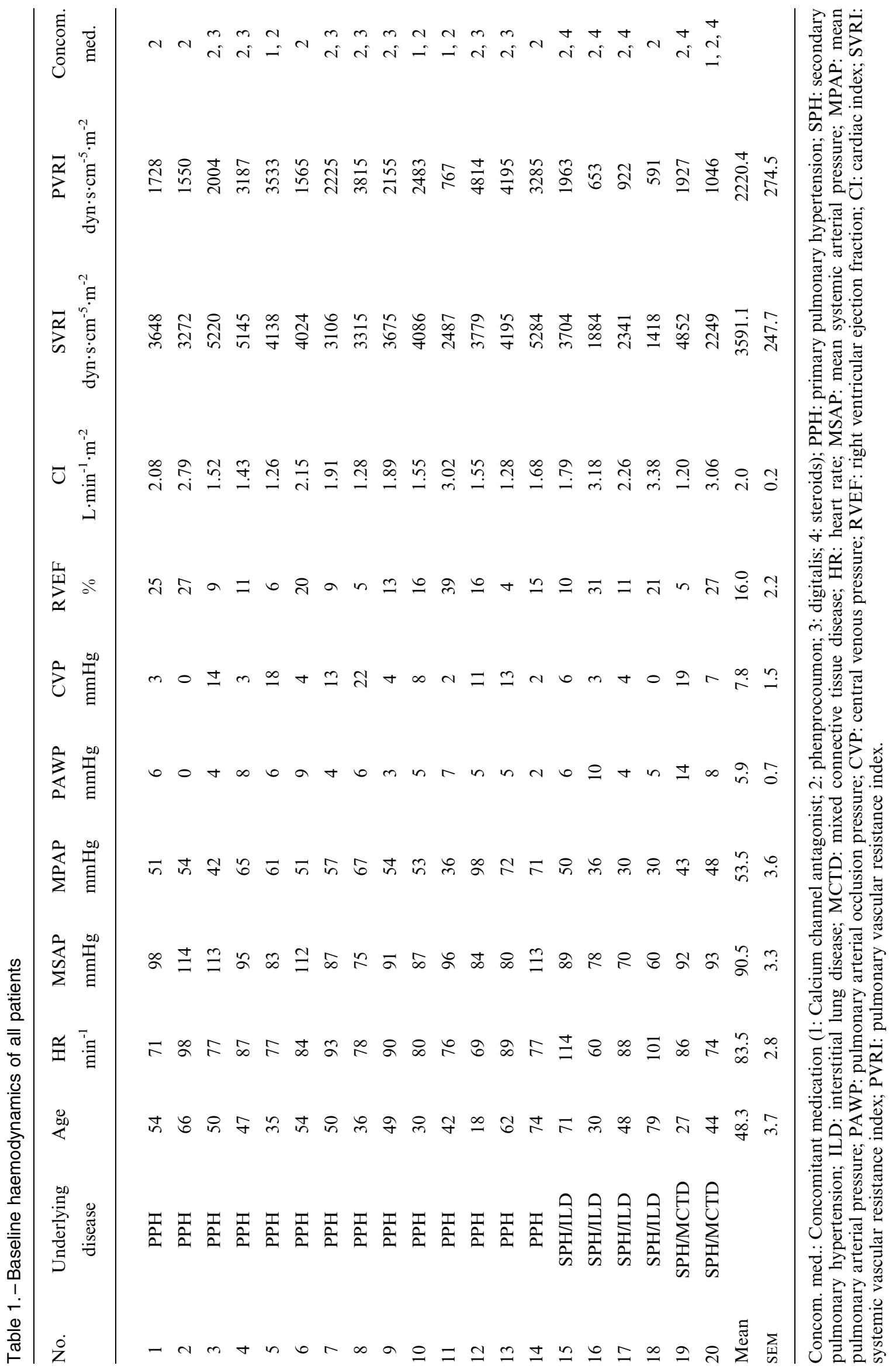


vasoreactivity between PPH and SPH patients. For each single parameter, the response to inhaled NO was considered significant if the 95\% $(p<0.05), 99 \%$ $(\mathrm{p}<0.01)$, or $99.9 \%(\mathrm{p}<0.001)$ confidence intervals of the pre-/post-difference did not overlap with zero. One way analysis of variance (ANOVA) was employed to determine differences in baseline parameters between the entire study population, NO responders and NO nonresponders.

\section{Results}

\section{Baseline conditions}

Twenty patients were enrolled into the study, all of which suffered from severe PH. In 14 patients the underlying disease was PPH and in the other six patients there were underlying or associated diseases (table 1). The patients were divided into two groups, depending on their haemodynamic responsiveness to NO inhalation (responders defined by a PVR index (PVRI) reduction of $\geqslant 20 \%$ ). NO responsiveness was detected in 13 out of 36 catheter tests $(36 \%)$, while in 23 tests, the patient exhibited no significant response. Of the 16 patients that were investigated twice, 10 were consistent nonresponders, two converted over a year period from moderate responders to nonresponders, and four were consistent responders. With regard to the first test only, six of the $14 \mathrm{PPH}$ patients were responders $(42 \%)$ and three of the six patients with $\mathrm{SPH}(50 \%)$ met the response criterium.

Haemodynamic parameters for the entire study population were as follows: heart rate (HR) $83.5 \pm 2.8 \mathrm{~min}^{-1}$, mean pulmonary artery pressure (MPAP) $53.5 \pm 3.6 \mathrm{mmHg}$, cardiac index (CI) $2.0 \pm$ $0.2 \mathrm{~L} \cdot \mathrm{min}^{-1} \cdot \mathrm{m}^{-2}$, PVRI 2,220.4 $274.5 \mathrm{dyn} \cdot \mathrm{s} \cdot \mathrm{cm}^{-5} \cdot \mathrm{m}^{-2}$, central venous pressure (CVP) $7.8 \pm 1.5 \mathrm{mmHg}$, and right ventricular ejection fraction (RVEF) $16.0 \pm 2.2 \%$ (table 1, fig. 1).

Plasma levels of cGMP in mixed venous and arterial blood samples were $14.0 \pm 1.7$ and $15.2 \pm 2.2 \mathrm{nM}$, respectively. Plasma ANP levels in mixed venous and arterial blood samples were $549.1 \pm 69.6$ and $514.4 \pm 64.6$ $\left(\mathrm{pg} \cdot \mathrm{mL}^{-1}\right)$, respectively. A clear correlation between mixed venous ANP and cGMP was noted (Pearson correlation $\mathrm{r}=0.47, \mathrm{p}<0.002)$. Strong correlations between pulmonary arterial cGMP and ANP levels, respectively, and PVRI were noted for the entire study population ( $\mathrm{r}=0.62, \mathrm{p}<0.0001$ (fig. $2 \mathrm{a}$ ), and $\mathrm{r}=0.66$, $\mathrm{p}<0.0001$, respectively).

After division into responders and nonresponders, the following haemodynamic values were noted in the subgroups. For NO responders: HR 79.5 $\pm 2.4 \mathrm{~min}^{-1}$,
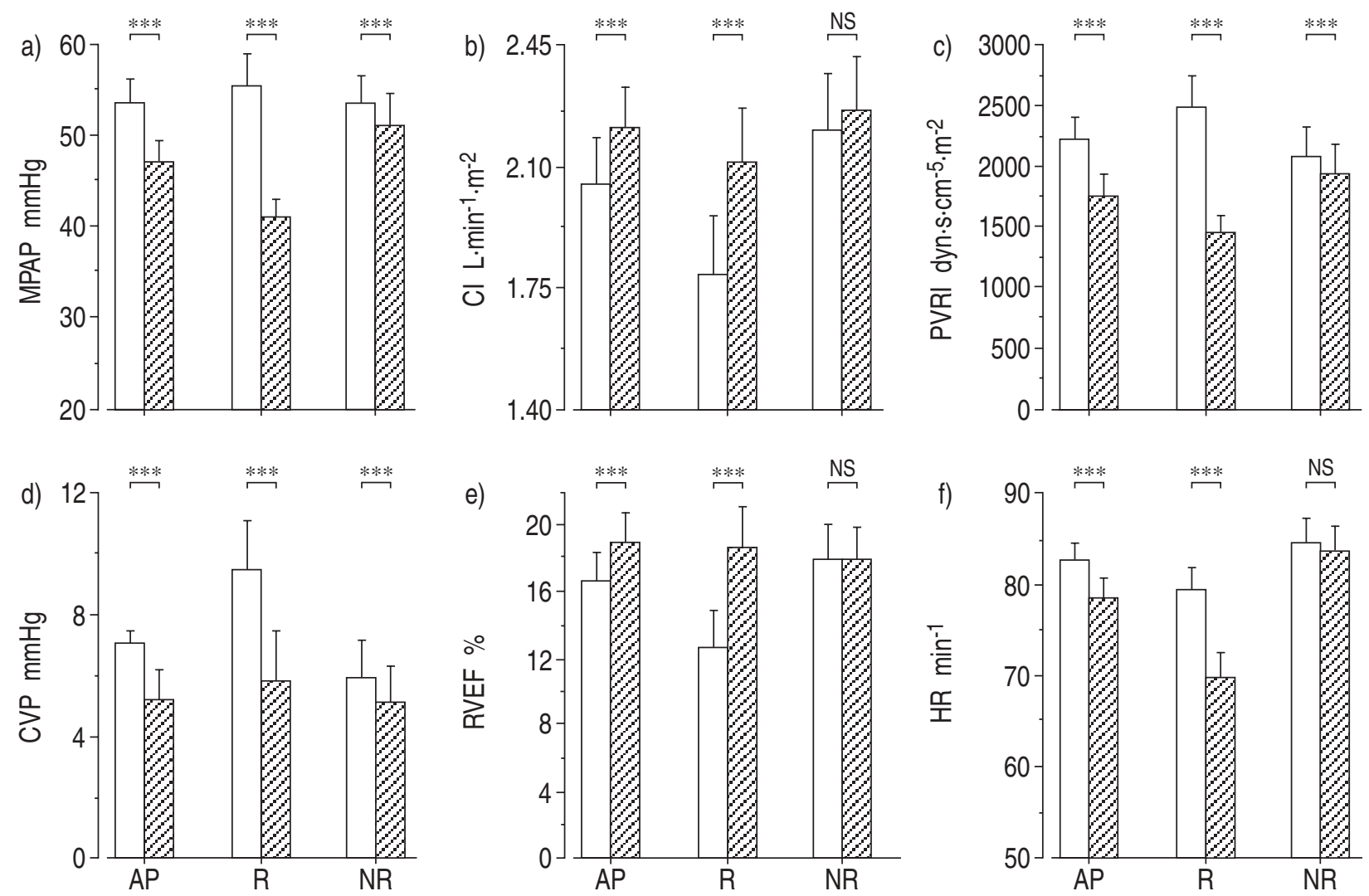

Fig. 1.-Haemodynamic variables (a) mean pulmonary artery pressure (MPAP); b) cardiac index (CI); c) pulmonary vascular resistance index (PVRI); d) central venous pressure (CVP); e) right ventricular ejection fraction (RVEF); f) heart rate (HR)) before and during nitric oxide (NO) inhalation. Baseline values of haemodynamics $(\square)$ and values obtained during NO inhalation ( $\mathbb{Z})$ are presented. Data are summarized for all catheter tests in all patients (AP; $n=36$ ) or are separately displayed for NO responders (R; defined by a PVR decrease of $\geqslant 20 \% ; n=13$ ) and NO nonresponders ( $N R ; n=23$ ) (mean \pm SEM). $* * *: p<0.001$, for differences between pre- and postexposure values. NS: nonsignificant. 

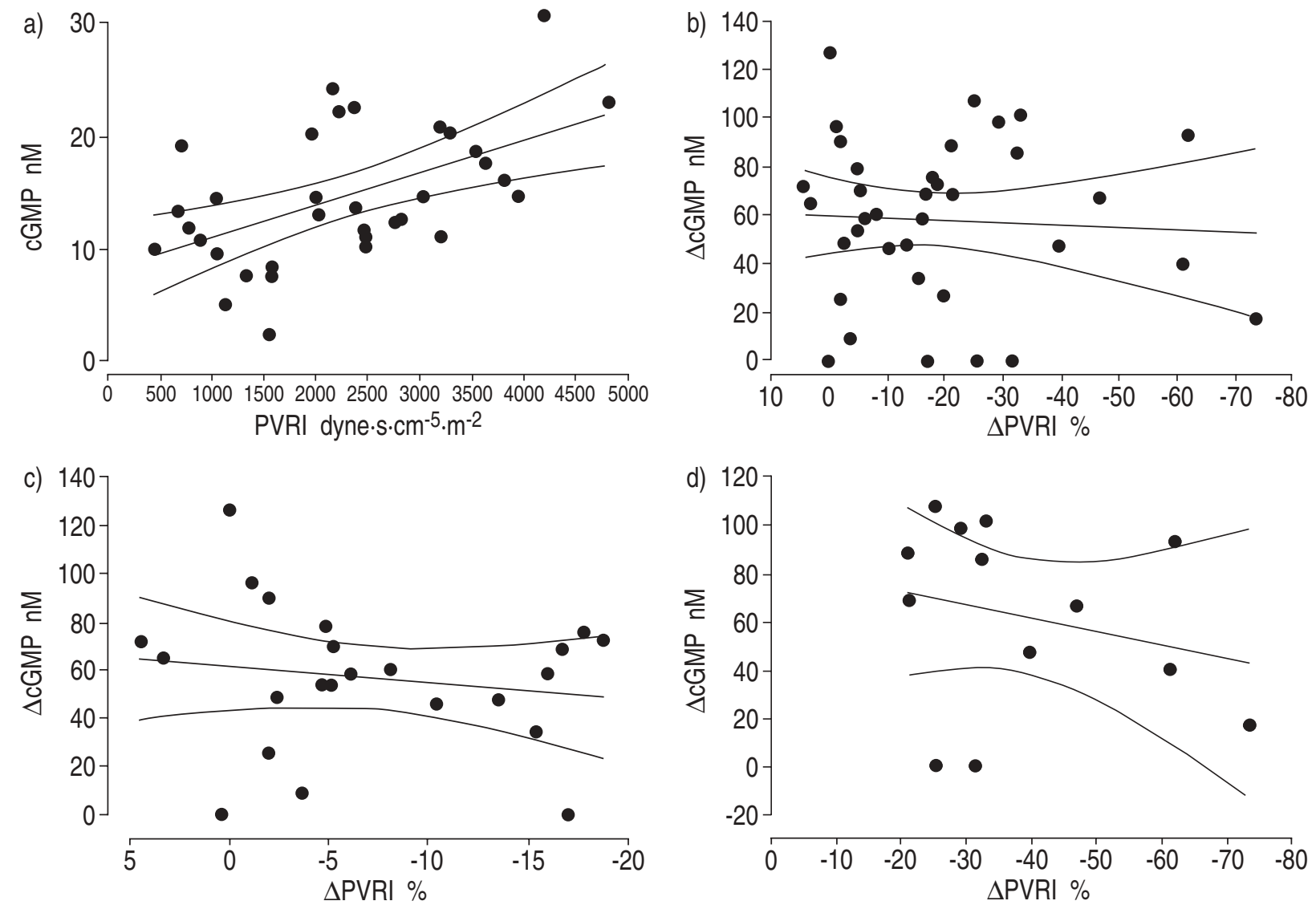

Fig. 2. - a) Correlation between pulmonary artery cyclic guanosine monophosphate (cGMP) levels and pulmonary vascular resistance index (PVRI) under baseline conditions. b-d) Correlation between changes in systemic arterial cGMP levels and changes in PVRI subsequent to nitric oxide (NO) inhalation. Values of all catheter tests in patients with primary pulmonary hypertension and secondary pulmonary hypertension $(\mathrm{n}=36 ; \mathrm{a}): \mathrm{r}=0.624, \mathrm{p}<0.0001 ; \mathrm{b}): \mathrm{r}=0.139$, nonsignificant $(\mathrm{NS}))$ and after separation into $\mathrm{NO}$ nonresponders $(\mathrm{n}=23 ; \mathrm{c}): \mathrm{r}=0.176$, NS) and NO responders ( $\mathrm{n}=13 ; \mathrm{d})$ : $\mathrm{r}=0.418, \mathrm{NS})$ are given. The linear regression curve and the $95 \%$ confidence interval are displayed.

MPAP $\quad 55.5 \pm 3.5 \mathrm{mmHg}, \quad$ CI $1.8 \pm 0.21 \mathrm{~L} \cdot \mathrm{min}^{-1} \cdot \mathrm{m}^{-2}$,

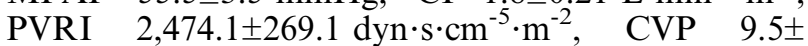
$1.7 \mathrm{mmHg}$, and RVEF $12.6 \pm 2.3 \%$. Plasma levels in pulmonary and systemic arterial blood for cGMP were $14.6 \pm 1.7$ and $15.9 \pm 2.4 \mathrm{nM}$, respectively. Plasma levels of ANP in pulmonary and systemic arterial blood were $678.7 \pm 128.7$ and $647 \pm 149.7 \mathrm{pg} \cdot \mathrm{mL}^{-1}$, respectively. For NO nonresponders: HR $84.5 \pm 2.5 \mathrm{~min}^{-1}$, MPAP $53.4 \pm 3.2 \mathrm{mmHg}, \quad$ CI $2.2 \pm 0.2 \mathrm{~L} \cdot \mathrm{min}^{-1} \cdot \mathrm{m}^{-2}$, PVRI 2,124.3 $\pm 240.8 \mathrm{dyn} \cdot \mathrm{s} \cdot \mathrm{cm}^{-5} \cdot \mathrm{m}^{-2}, \quad$ CVP 5.9 $1.3 \mathrm{mmHg}$, and RVEF $17.9 \pm 2.1 \mathrm{mmHg}$. Plasma levels of cGMP in pulmonary and systemic arterial blood were $12.0 \pm 1.2$ and $13.2 \pm 1.5 \mathrm{nM}$, respectively. Plasma levels of ANP in pulmonary and systemic arterial blood were $478.5 \pm 79.9$ and $444.6 \pm$ $57.4 \mathrm{pg} \cdot \mathrm{mL}^{-1}$, respectively.

No significant differences in the baseline parameters (haemodynamics and cGMP or ANP levels) of the entire study population, NO responders and NO nonresponders were detected through analysis of variance.

\section{Nitric oxide inhalation}

MPAP was statistically significantly reduced in response to $\mathrm{NO}$ inhalation in all groups of patients $(-6.8 \pm 1.6 \%,-14.8 \pm 3.5 \%$, and $-2.4 \pm 0.6 \%$ for the entire study population, the responders, and the nonresponders, respectively $(\mathrm{p}<0.001))$, whereas CI and RVEF significantly increased only in the entire population and responder subgroup $(\mathrm{p}<0.001$; fig. 1). As anticipated due to the definition of the subgroups, the PVRI decrease in response to NO was particularly prominent for the responder group. This was also true for the decrease in CVP in response to the gaseous vasodilator.

After NO inhalation, there was a huge and highly significant elevation of cGMP levels in both pulmonary arterial and systemic arterial blood samples in all groups of patients $(p<0.001$; fig. 3$)$. A marked transpulmonary cGMP gradient, which was not detectable under baseline conditions, also became evident (fig. 3). However, both the pre-/post-NO differences in pulmonary and systemic arterial cGMP levels and the transpulmonary cGMP gradient did not differ between the NO responder and nonresponder groups. Moreover, when correlating the decrease in PVRI with the increase in cGMP levels in response to NO, no significant relationship was noted for the entire group of patients or for the subgroups of responders and nonresponders (fig. $2 b-d$ ). The same was true for other parameters of right ventricular loading and performance, e.g. RVEF, CVP, venous oxygen 

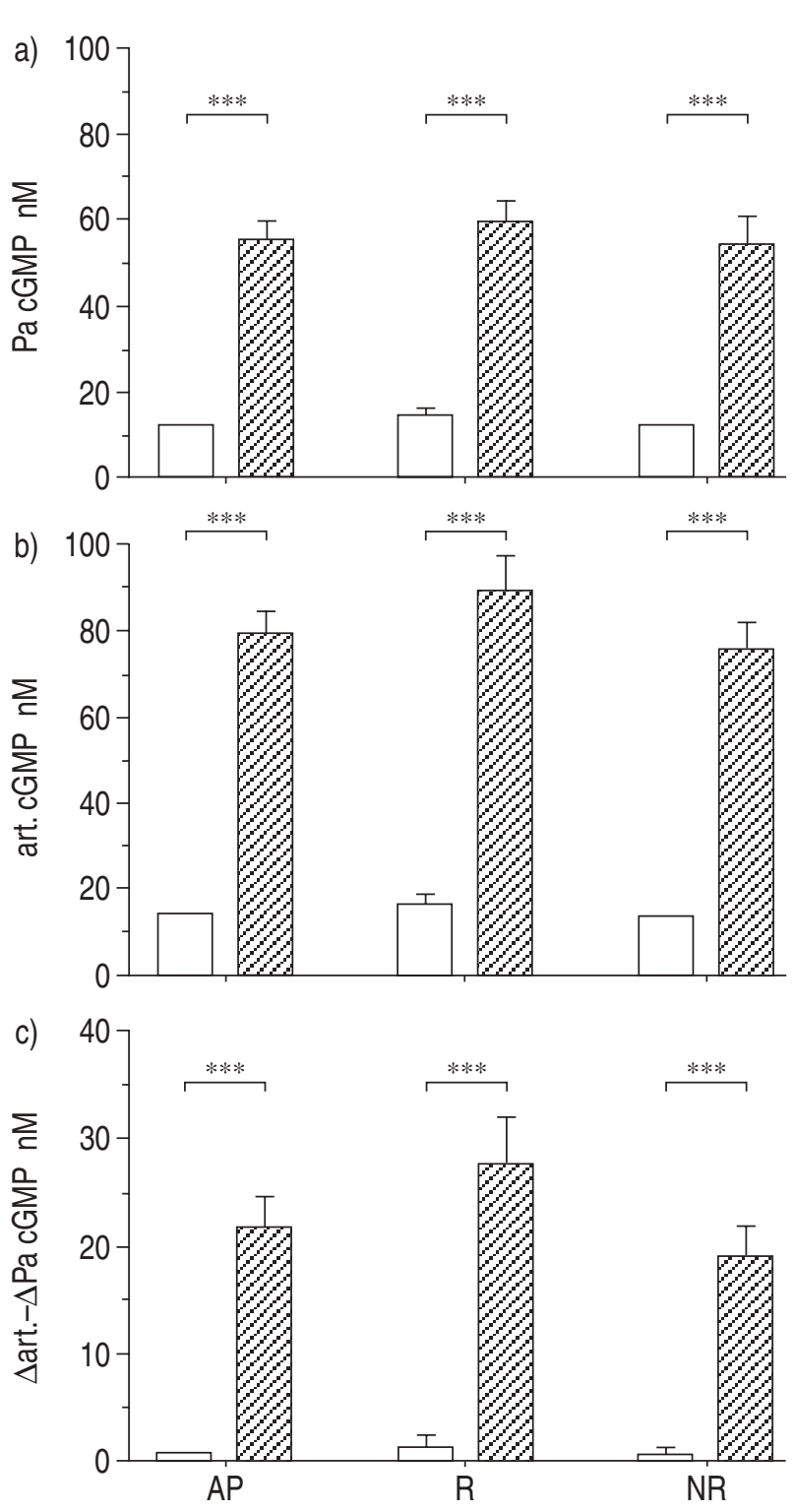

Fig. 3.-a) Changes in pulmonary arterial $(\mathrm{Pa})$ cyclic guanosine monophosphate (cGMP) (Pa cGMP) levels, b) systemic arterial (art.) cGMP levels (art. cGMP) and c) the transpulmonary cGMP gradient ( $\triangle$ art.- $\triangle \mathrm{Pa}$ cGMP), in response to nitric oxide (NO) inhalation. Baseline values $(\square)$ and values during NO inhalation $(\mathbb{Z})$ are presented (mean \pm SEM). Data are given for all patients (AP; $n=36$ ), or are displayed separately for $\mathrm{NO}$ responders (R; $\mathrm{n}=13$ ) and NO nonresponders (NR; $\mathrm{n}=23)$. ${ }^{* * *}: \mathrm{p}<0.001$.

saturation, CI (data not given in detail). However, a significant reduction of mixed venous ANP levels was only observed in responders after NO inhalation $\left(-129.5 \pm 59.6 \mathrm{pg} \cdot \mathrm{mL}^{-1}, \mathrm{p}<0.05\right)$.

The analysis of all data with respect to $\mathrm{PPH}$ versus SPH patients, did not reveal any significant differences between their baseline haemodynamics, cGMP levels, and responsiveness to NO inhalation (unpaired t-test, nonsignificant). Furthermore, when analysing only the PPH patients with respect to their NO responsiveness, the findings did not differ from those obtained when the entire group of patients were analysed.

\section{Discussion}

In patients with severe precapillary $\mathrm{PH}$, the majority of whom were classified with PPH, a significant correlation between baseline plasma cGMP and ANP levels, and the severity of disease was noted. In response to NO inhalation, a pronounced increase in central venous and arterial cGMP levels occurred, accompanied by the appearance of a marked transpulmonary cGMP gradient, strongly suggestive of enhanced lung cGMP synthesis during NO inhalation. However, the magnitude of the NOelicited cGMP response did not discriminate between those patients displaying a marked pulmonary vasorelaxation in response to this agent and those classified as nonresponders in terms of acute lung vasodilatation.

Pharmacological challenge with inhalative NO, a short-acting vasodilatory agent possessing selectivity for the pulmonary circulation, is now largely accepted as a convenient tool to detect patients with a good acute responsiveness to vasodilators [5, 19]. It has previously been shown that haemodynamic responsiveness to inhaled $\mathrm{NO}$ is a good predictor for a beneficial response to high-dose oral calcium channel blockers $[19,20]$. Long-term treatment with these substances should be restricted to these acute responders, since clinical benefit is not to be expected in nonresponders [21]. In this study of 20 patients with severe precapillary $\mathrm{PH}$, including 14 with $\mathrm{PPH}$ and six similarly affected patients, haemodynamic response to NO was assessed during 36 right heart catheter tests. In 13 of the catheter tests, a reduction in PVR of $>20 \%$ was noted and chosen to define responders, whereas nonresponsiveness was noted in 23 of the catheter tests, resulting in a responder rate of $\sim 30 \%$, which is in line with the data of previous studies addressing this issue (20-33\%) [1]. According to a recent report, a responder rate of $\sim 80 \%$ was noted in $\mathrm{PPH}$ patients [22], but this rate is far in excess of that detected in studies from other centres. The variation is, in part, attributable to the different definitions being employed to characterize NO responsiveness and to the different percentage of patients with SPH being included in the trials.

When addressing the question "which variables might discriminate between NO responders and nonresponders?", PPH and SPH patients were treated as one common collective. It was felt that this procedure was justified, as PPH and SPH patients did not differ significantly with respect to baseline haemodynamics, baseline plasma cGMP and ANP levels and NO responder rate (six out of 14 patients in the $\mathrm{PPH}$ group were responders and three out of six patients in the SPH group were responders).

A number of factors may be relevant when considering NO responsiveness. These include the severity of haemodynamic abnormalities, the baseline levels of cGMP, and the increment of plasma cGMP levels and establishment of a significant transpulmonary cGMP gradient in response to NO. Each of these factors will now be considered in turn. 


\section{Severity of haemodynamic abnormalities}

No significant association between pulmonary artery pressure/resistance or the severity of right heart decompensation and the state of NO responsiveness was noted.

\section{Baseline levels of cyclic guanosine monophosphate}

Interestingly, a significant correlation between the severity of PH and baseline cGMP levels was observed. This finding suggests that enhanced cGMP formation may be part of a negative feedback mechanism, counteracting the increased right ventricular afterload in both PPH and SPH. As cGMP synthesis may be triggered by various stimuli, including $\mathrm{NO}$ (soluble guanylate cyclase) and ANPs (particulate guanylate cyclase), different mechanisms may underlie the increase in plasma cGMP levels in severe PH. The ANP levels in the present study subjects were dramatically elevated compared to normal subjects. There was a clear correlation between cGMP levels and ANP in mixed venous blood at baseline, as the level of both was also correlated to parameters of right ventricular load. This observation is in line with the finding that circulating ANP and brain natriuretic peptide (BNP) are markedly enhanced in severe $\mathrm{PH}$ and even considered to be of prognostic relevance for the disease $[23,24]$. With regard to lung vascular NO formation, both enhanced and reduced expression of endothelial NO synthase activity has been reported for $\mathrm{PPH}[25,26]$. The present findings, which show a correlation between plasma cGMP values and the severity of $\mathrm{PH}$, correspond with the previous observation that levels of urinary cGMP are associated with the severity of disease in patients with PPH [11].

Increment of plasma cyclic guanosine monophosphate levels and establishment of a significant transpulmonary cyclic guanosine monophosphate gradient in response to nitric oxide

Inhalation of NO caused an increase in both pulmonary and systemic arterial cGMP levels in nearly all patients. Moreover, a marked transpulmonary cGMP gradient appeared under these conditions, strongly indicating substantial intrapulmonary cGMP production in response to NO inhalation. However, this gradient was not correlated with the haemodynamic response to $\mathrm{NO}$ and, in particular, did not discriminate between responders and nonresponders. Interestingly, similar observations were made in patients with acute lung failure [10]. In these mechanically-ventilated patients, "responders" were defined by an NO-induced improvement in arterial oxygenation, caused by selective vasodilatation in well-ventilated lung areas, but no correlation between the gas exchange response and the increment in plasma cGMP levels during NO inhalation was detected. One possible explanation for the lack of association between NO-induced pulmonary vasodilatation and increase in blood cGMP levels may be the fact that plasma cGMP stems from various cell types, whereas a highly compartmentalized NO/cGMP effect may be responsible for the lung vasodilatory effect. Moreover, ANP levels in mixed venous blood were reduced in responders after NO inhalation, indicating a reduced release of ANP under circumstances of right ventricular (and atrial) relief. However, no significant correlation could be observed with the extent of PVR reduction, which might be attributable to the limited number of patients in the responder group. The reduction of ANP levels in patients displaying a favourable vasoreactivity may thus account for reduced "endogenous" NO production and counteract the elevation of "exogenously" (NO-driven) cGMP production. In addition, mechanisms of signal transduction distal to cGMP production may be responsible for the observed phenomenon. In vitro studies have demonstrated that cGMP relaxes vascular smooth muscle cells by activation of potassium channels via a cGMP dependent protein kinase [27, 28]. Differences in the responsiveness of the downstream signalling cascade, rather than differences in the cGMP secretion in response to NO, may be the factors discriminating between $\mathrm{NO}$ responders and nonresponders. Finally, possible differences in local phosphodiesterase (PDE) activity cannot be disregarded. It is known that regulation of PDE-5 (cGMP hydrolyzing phosphodiesterase) influences vascular tone and vessel proliferation during lung development [29]. Furthermore, in experimental perinatal PH, PDE-5 activity was demonstrated to play a pivotal role in the mediation of increased pulmonary resistance [30]. In addition to the magnitude of NOinduced cGMP synthesis, regional PDE-5 activity might thus contribute to the vasorelaxant response to this gaseous agent.

In conclusion, baseline plasma cyclic guanosine monophosphate levels are significantly correlated with the severity of both primary and secondary pulmonary hypertension. However, the nitric oxide-induced increment in cyclic guanosine monophosphate plasma levels and the magnitude of the transpulmonary cyclic guanosine monophosphate gradient occurring during nitric oxide inhalation, are not linked with the lung vasodilatory response to this agent. Measurement of changes in cyclic guanosine monophosphate plasma levels occurring during nitric oxide inhalation may not, therefore, be a substitute for right heart catheterization when testing patients with primary and secondary pulmonary hypertension for nitric oxide responsiveness.

\footnotetext{
Acknowledgements. The authors would like to thank C. Traber-Ferdinand and Z. Erdogan for their excellent technical assistance, and N. Katz (Clinical chemistry, Justus-Liebig-University, Giessen) for supporting the cGMP measurements.
}

\section{References}

1. Gaine SP, Rubin LJ. Primary pulmonary hypertension. Lancet 1998; 352: 719-725. 
2. D'Alonzo GE, Barst RJ, Ayres SM, et al. Survival in patients with primary pulmonary hypertension. Results from a national prospective registry. Ann Intern Med 1991; 115: 343-349.

3. Rich S, Kaufmann E, Levy PS. The effect of high doses of calcium-channel blockers on survival in primary pulmonary hypertension. $N$ Engl $\mathrm{J}$ Med 1992; 327: 76-81.

4. Sitbon $\mathrm{O}$, Humbert $\mathrm{M}$, Jagot JL, et al. Inhaled nitric oxide as a screening agent for safely identifying responders to oral calcium-channel blockers in primary pulmonary hypertension. Eur Respir J 1998; 12: 265-270.

5. Jolliet P, Bulpa P, Thorens JB, Ritz M, Chevrolet JC. Nitric oxide and prostacyclin as test agents of vasoreactivity in severe precapillary pulmonary hypertension: predictive ability and consequences on haemodynamics and gas exchange. Thorax 1997; 52: 369-372.

6. Ricciardi MJ, Knight BP, Martinez FJ, Rubenfire M. Inhaled nitric oxide in primary pulmonary hypertension: a safe and effective agent for predicting response to nifedipine. J Am Coll Cardiol 1998; 32: 1068-1073.

7. Lucas KA, Pitari GM, Kazerounian S, et al. Guanylyl cyclases and signaling by cyclic GMP. Pharmacol Rev 2000; 52: 375-414.

8. Waldman SA, Murad F. Cyclic GMP synthesis and function. Pharmacol Rev 1987; 39: 163-196.

9. Horstman DJ, McCall DA, Frank DU, Rich GF. Inhaled nitric oxide and nifedipine have similar effects on lung cGMP levels in rats. Anesth Analg 1999; 89: 932-937.

10. Zwissler B, Kemming G, Merkel M, et al. Response to inhaled nitric oxide (NO) is not associated with changes of plasma cGMP levels in patients with acute lung injury. Eur J Med Res 1999; 4: 463-467.

11. Bogdan M, Humbert M, Francoual J, et al. Urinary cGMP concentrations in severe primary pulmonary hypertension. Thorax 1998; 53: 1059-1062.

12. Cargill RI, Lipworth BJ. Pulmonary vasorelaxant activity of atrial natriuretic peptide and brain natriuretic peptide in humans. Thorax 1995; 50: 183-185.

13. Itoh $\mathrm{H}$, Pratt RE, Dzau VJ. Atrial natriuretic polypeptide inhibits hypertrophy of vascular smooth muscle cells. J Clin Invest 1990; 86: 1690-1697.

14. Winter RJ, Zhao L, Krausz T, Hughes JM. Neutral endopeptidase 24.11 inhibition reduces pulmonary vascular remodeling in rats exposed to chronic hypoxia. Am Rev Respir Dis 1991; 144: 1342-1346.

15. Pedram A, Razandi M, Hu RM, Levin ER. Vasoactive peptides modulate vascular endothelial cell growth factor production and endothelial cell proliferation and invasion. J Biol Chem 1997; 272: $17097-$ 17103.

16. Levin ER, Gardner DG, Samson WK. Natriuretic peptides. N Engl J Med 1998; 339: 321-328.

17. Rich S, ed. Primary Pulmonary Hypertension: Executive Summary from the World Symposium - Primary
Pulmonary Hypertension. www.who.int/ncd/cvd/pph. html. Date accessed: 1998.

18. Olschewski H, Ghofrani HA, Schmehl T, et al. Inhaled iloprost to treat severe pulmonary hypertension. An uncontrolled trial. German PPH Study Group. Ann Intern Med 2000; 132: 435-443.

19. Sitbon O, Brenot F, Denjean A, et al. Inhaled nitric oxide as a screening vasodilator agent in primary pulmonary hypertension. A dose-response study and comparison with prostacyclin. Am J Respir Crit Care Med 1995; 151: 384-389.

20. Galie N, Ussia G, Passarelli P, Parlangeli R, Branzi A, Magnani B. Role of pharmacologic tests in the treatment of primary pulmonary hypertension. $\mathrm{Am}$ J Cardiol 1995; 75: 55A-62A.

21. Gaine S. Pulmonary hypertension. JAMA 2000; 284 : 3160-3168.

22. Krasuski RA, Warner JJ, Wang A, Harrison JK, Tapson VF, Bashore TM. Inhaled nitric oxide selectively dilates pulmonary vasculature in adult patients with pulmonary hypertension, irrespective of etiology. J Am Coll Cardiol 2000; 36: 2204-2211.

23. Morice AH, Pepke-Zaba J, Brown MJ, Thomas PS, Higenbottam TW. Atrial natriuretic peptide in primary pulmonary hypertension. Eur Respir J 1990; 3: 910-913.

24. Nagaya N, Nishikimi T, Uematsu M, et al. Plasma brain natriuretic peptide as a prognostic indicator in patients with primary pulmonary hypertension. Circulation 2000; 102: 865-870.

25. Mason NA, Springall DR, Burke M, et al. High expression of endothelial nitric oxide synthase in plexiform lesions of pulmonary hypertension. $J$ Pathol 1998; 185: 313-318.

26. Giaid A, Saleh D. Reduced expression of endothelial nitric oxide synthase in the lungs of patients with pulmonary hypertension. N Engl J Med 1995; 333: 214-221.

27. Archer SL, Huang JM, Hampl V, Nelson DP, Shultz PJ, Weir EK. Nitric oxide and cGMP cause vasorelaxation by activation of a charybdotoxin-sensitive K channel by cGMP-dependent protein kinase. Proc Natl Acad Sci USA 1994; 91: 7583-7587.

28. Hampl V, Huang JM, Weir EK, Archer SL. Activation of the cGMP-dependent protein kinase mimics the stimulatory effect of nitric oxide and cGMP on calcium-gated potassium channels. Physiol Res 1995; 44: 39-44.

29. Hanson KA, Burns F, Rybalkin SD, Miller JW, Beavo J, Clarke WR. Developmental changes in lung cGMP phosphodiesterase-5 activity, protein, and message. Am J Respir Crit Care Med 1998; 158: 279 288.

30. Hanson KA, Ziegler JW, Rybalkin SD, Miller JW, Abman SH, Clarke WR. Chronic pulmonary hypertension increases fetal lung cGMP phosphodiesterase activity. Am J Physiol 1998; 275: L931-L941. 